\title{
A variable step-size gradient adaptive lattice algorithm for multiple sinusoidal interference cancelation
}

Seong-woo Kim¹, Young-cheol Park ${ }^{2}$ and Dae Hee Youn ${ }^{1 *}$

\begin{abstract}
In passive sonar, adaptive algorithms can be used to cancel strong sinusoidal self-interferences. In order to correctly recover low-power target signals during the early stages of processing, these adaptive algorithms must provide fast convergence and, at the same time, narrow notches at the frequencies of the sinusoids. In this respect, the gradient adaptive lattice (GAL) algorithm is a very attractive choice. However, the GAL algorithm with a constant step-size parameter has to compromise between the convergence rate and notch bandwidths. Therefore, in this article, we propose a variable step-size scheme for the GAL algorithm that can achieve both a fast convergence rate and narrow notches. Simulation results demonstrate the efficiency of the proposed algorithm compared to both the conventional GAL algorithm and transversal adaptive filter combined with the variable step-size scheme.
\end{abstract}

Keywords: Adaptive noise cancelation (ANC), Adaptive filters, Gradient adaptive lattice (GAL) algorithm, Variable step-size gradient adaptive lattice (VSS-GAL) algorithm

\section{Introduction}

The performance of passive sonar is easily degraded by strong self-interference originating from the machinery of its own ship [1]. To cancel this self-interference composed of multiple sinusoids, adaptive noise cancelation (ANC) techniques can be used [2]. An ANC algorithm should be able to form narrow notches in order to filter out self-interference without distorting the low-power target signal in a passive sonar signal [3]. Among the various adaptive algorithms, the normalized least mean square (NLMS) algorithm is most widely used due to its computational simplicity and ease of implementation [4]. However, the least mean square (LMS)-type transversal adaptive filters produce notch bandwidths proportional to interference amplitudes [3,5], which can result in the distortion of the target signal in passive sonar especially when the interference has strong amplitude.

The gradient adaptive lattice (GAL) algorithm has also been widely used for ANC [6,7]. Unlike the LMS-type

\footnotetext{
*Correspondence: dhyoun@yonsei.ac.kr

${ }^{1}$ School of Electrical and Electronic Engineering, Yonsei University, Seoul 120-749, South Korea
}

Full list of author information is available at the end of the article transversal filters, it produces notches whose bandwidths are independent of interference amplitudes [5]. Thus, the GAL algorithm is more desirable than the LMStype transversal filter in terms of the distortion in the recovered target signal. However, the GAL algorithm has the property that the step-size parameter reflects a tradeoff between the convergence rate and the notch bandwidths, and thus, relatively wide notch bandwidths should be allowed to achieve fast convergence rate. To achieve both fast convergence rate and narrow notch bandwidth, a variable step-size (VSS) scheme can be considered. Many VSS schemes have been developed mainly for the transversal filters employing the LMS and affine projection algorithms [8-14]. However, these VSS schemes are not directly applicable to the GAL algorithm due to different structure and convergence behaviors. Previously, a variable step-size filtered-x GAL (VSS-FxGAL) algorithm was presented by the authors to obtain a fast adaptive algorithm for active noise control applications [15]. To estimate parameters for the step-size control in practical situations, [15] uses the assumption that the system is in a converged state, which, however, can be problematic especially when the system is in a time-varying environment.

\section{焦 Springer}

(c) 2013 Kim et al: licensee Springer. This is an Open Access article distributed under the terms of the Creative Commons Attribution License (http://creativecommons.org/licenses/by/2.0), which permits unrestricted use, distribution, and reproduction in any medium, provided the original work is properly cited. 
In this article, we revise the previous VSS-FxGAL algorithm to make it suitable for the cancelation of selfinterference in passive sonar. The proposed VSS scheme takes into account the fact that the target signal needs to be preserved after canceling the strong self-interference. Thus, the VSS scheme is developed from a condition that the target signal power is recovered at the error output. As a result, the proposed algorithm achieves both fast convergence and narrow notches at the sinusoid frequencies. Convergence analysis in the mean square sense is presented to prove the stability and steady-state performance. Simulation results corroborate the analyses in various environments.

The rest of the article is organized as follows. Section 2 starts with a presentation of the ANC configuration. Then, the classical GAL algorithm is introduced, followed by the derivation and analysis of the proposed VSS-GAL algorithm. Section 3 presents the parameter estimation for real implementation. The simulation results are presented in Section 4, comparing the proposed VSS-GAL algorithm from the GAL algorithm and the variable stepsize NLMS (VSS-NLMS) algorithm. Finally, in Section 5, the main results of this work are discussed, and the conclusions are drawn.

\section{Variable step-size GAL algorithm}

The structure of the GAL noise cancelation algorithm is shown in Figure 1. The target signal $s(n)$ is corrupted by the uncorrelated interference $v_{1}(n)$. The purpose of the GAL algorithm is to adaptively match the interference $v_{1}(n)$ in the primary signal $d(n)=s(n)+v_{1}(n)$ by linearly combining the backward prediction errors obtained from the reference signal $v_{2}(n)$.

\subsection{GAL algorithm}

Consider an $M$ th-order lattice predictor specified by the recursive equations [6]:

$$
\begin{aligned}
& f_{m+1}(n)=f_{m}(n)-\kappa_{m}(n-1) b_{m}(n-1) \\
& b_{m+1}(n)=b_{m}(n-1)-\kappa_{m}(n-1) f_{m}(n), 0 \leq m<M-1,
\end{aligned}
$$

where $f_{m}(n)$ and $b_{m}(n)$ denote the $m$ th stage forward and backward prediction errors at time $n$, respectively, and $\kappa_{m}(n)$ is the reflection coefficient. The backward prediction errors are orthogonal to each other as

$$
E\left\{b_{m}(n) b_{l}(n)\right\}=\left\{\begin{array}{c}
\eta_{m}(n), m=l \\
0, m \neq l
\end{array},\right.
$$

where $\eta_{m}(n)=E\left\{b_{m}^{2}(n)\right\}$ denotes the power of the $m$ th stage backward prediction error. It can be recursively estimated using the single-pole low-pass filter as $\hat{\eta}_{m}(n)=$ $\lambda \hat{\eta}_{m}(n-1)+(1-\lambda) b_{m}^{2}(n)$, where $\lambda$ is a smoothing factor. In the classical GAL algorithm [6], the $m$ th stage reflection coefficient is updated as

$$
\begin{aligned}
\kappa_{m}(n)= & \kappa_{m}(n-1)+\frac{\mu}{\xi_{m}(n)} \\
& \times\left[f_{m+1}(n) b_{m}(n-1)+f_{m}(n) b_{m+1}(n)\right]
\end{aligned}
$$

where $\mu$ is the step-size parameter and $\xi_{m}(n)=$ $E\left\{b_{m}^{2}(n-1)+f_{m}^{2}(n)\right\}$ is the power of both the $m$ th stage forward and delayed backward prediction errors, which can also be recursively estimated. The update equation for the $m$ th stage regression coefficient $w_{m}(n)$ is expressed as

$$
\begin{aligned}
& w_{m}(n)=w_{m}(n-1)+\frac{\mu}{\eta_{m}(n)} b_{m}(n) e_{m}(n) \\
& e_{m}(n)=e_{m-1}(n)-w_{m}(n-1) b_{m}(n),
\end{aligned}
$$

where $e_{m}(n)$ is the $m$ the stage error signal and $e_{-1}(n)=$ $d(n)$ is the initial error signal.

\subsection{Variable step-size GAL algorithm}

To derive the variable step-size GAL algorithm, we assume that the adaptive filter has converged to a certain degree $[9,15]$. Then, due to the orthogonality of the backward prediction errors [6], we can establish the approximation:

$$
\begin{aligned}
E\left\{b_{m}(n) e_{m}(n)\right\}= & E\left\{b_{m}(n) d(n)\right\} \\
& -\sum_{i=0}^{m} E\left\{w_{i}(n-1) b_{m}(n) b_{i}(n)\right\} \\
\approx & E\left\{b_{m}(n) d(n)\right\} \\
& -\sum_{i=0}^{M-1} E\left\{w_{i}(n-1) b_{m}(n) b_{i}(n)\right\} \\
= & E\left\{b_{m}(n) e_{M-1}(n)\right\},
\end{aligned}
$$

where $e_{M-1}(n)$ denotes the $(M-1)$ th stage error signal. Using this approximation, the update equation in Eq. (5) can be rewritten in vector notations as

$$
\begin{aligned}
& \mathbf{w}(n)=\mathbf{w}(n-1)+\mu(n) \Sigma^{-1}(n) \mathbf{b}(n) e_{M-1}(n) \\
& e_{M-1}(n)=d(n)-\mathbf{w}^{T}(n-1) \mathbf{b}(n),
\end{aligned}
$$

where $\mathbf{w}(n)=\left[w_{0}(n), \ldots, w_{M-1}(n)\right]^{T}$ and $\mathbf{b}(n)=$ $\left[b_{0}(n), \ldots, b_{M-1}(n)\right]^{T}$ are the regression coefficients vector and the backward prediction errors vector, respectively, and $\Sigma(n)=\operatorname{diag}\left\{\eta_{0}(n), \ldots, \eta_{M-1}(n)\right\}$ is a diagonal matrix. The positive scalar $\mu(n)$ in Eq. (8) denotes the variable step-size parameter. 


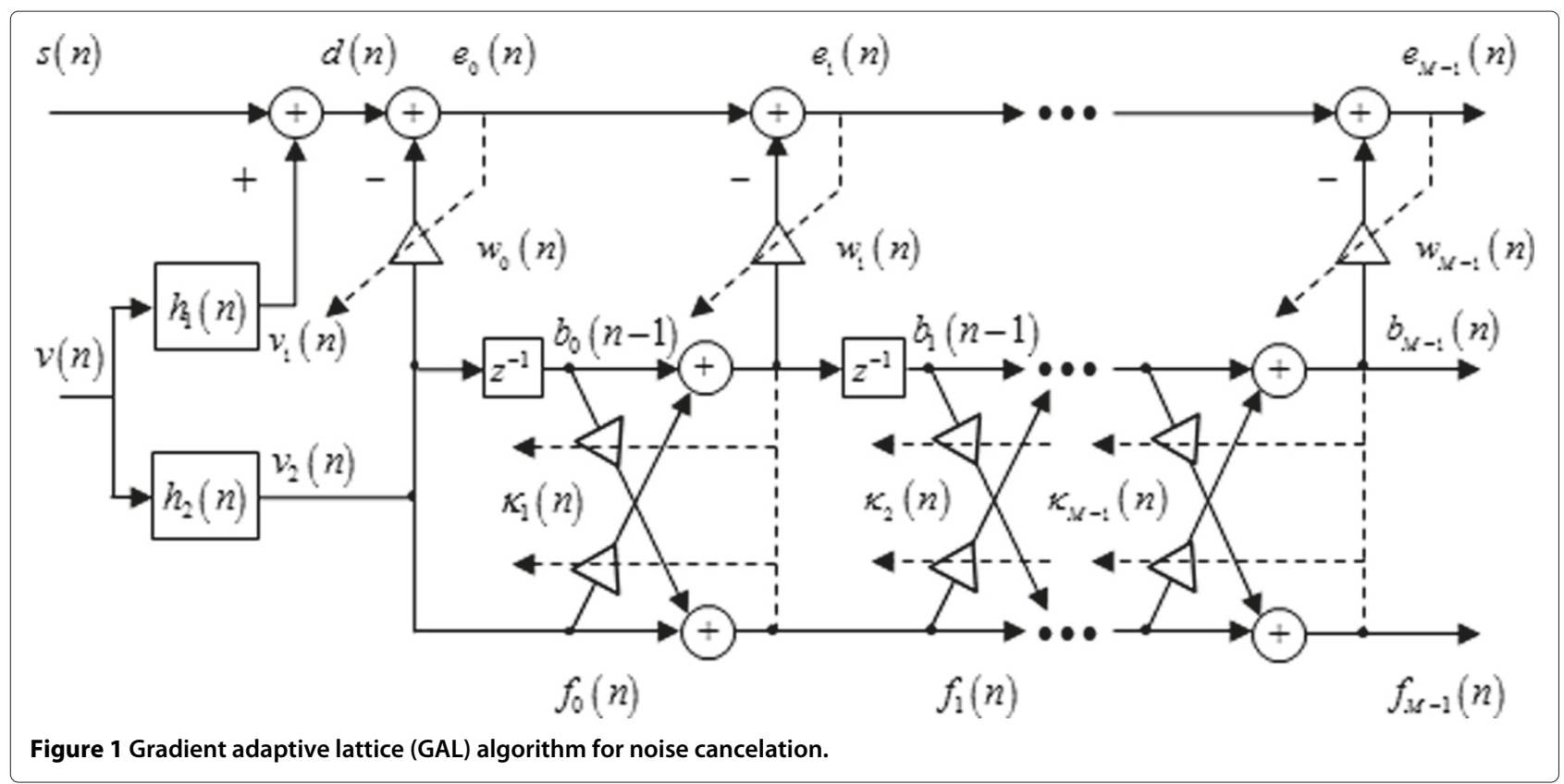

Using the regression coefficients at time $n$, the $a$ posteriori error signal can be defined as

$$
\varepsilon(n)=d(n)-\mathbf{w}^{T}(n) \mathbf{b}(n) .
$$

Substituting Eq. (8) into Eq. (10), we have

$$
\varepsilon(n)=\left[1-\mu(n) \mathbf{b}^{T}(n) \Sigma^{-1}(n) \mathbf{b}(n)\right] e_{M-1}(n) .
$$

The target signal in passive sonar should be recovered with minimum distortion at the filter output. To this end, we can find a variable step-size parameter $\mu(n)$ that satisfies the following condition:

$$
\sigma_{\varepsilon}^{2}(n)=\sigma_{s}^{2}(n), \forall n,
$$

where $\sigma_{\varepsilon}^{2}(n)=E\left\{\varepsilon^{2}(n)\right\}$ and $\sigma_{s}^{2}(n)=E\left\{s^{2}(n)\right\}$ are the powers of the a posteriori error signal and target signal, respectively. Thus, using the condition in Eq. (12), the target signal power is recovered at the a posteriori error signal. Now, squaring Eq. (11) and taking the
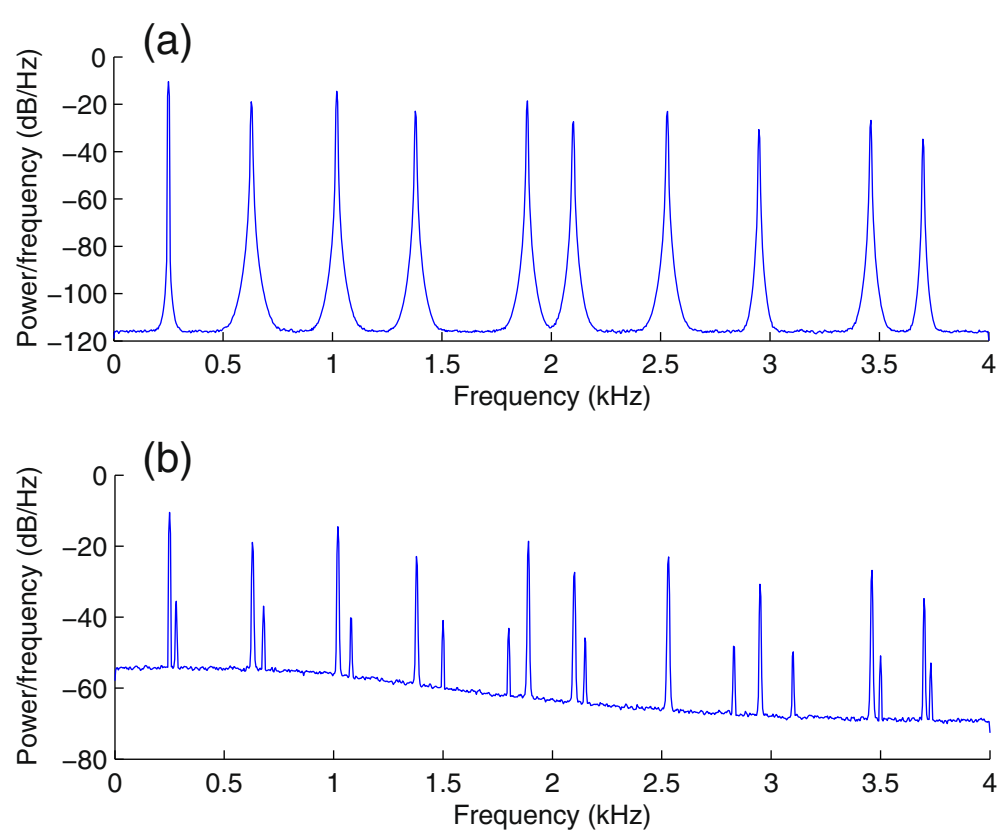

Figure 2 Power spectral densities of (a) the reference signal and (b) primary signal (target + interference). 
expectations under the independence assumption [16], we obtain

$$
\begin{aligned}
{[1} & -2 E\left\{\mathbf{b}^{T}(n) \Sigma^{-1}(n) \mathbf{b}(n)\right\} \mu(n) \\
& \left.+E\left\{\mathbf{b}^{T}(n) \Sigma^{-1}(n) \mathbf{b}(n) \mathbf{b}^{T}(n) \Sigma^{-1}(n) \mathbf{b}(n)\right\} \mu^{2}(n)\right] \\
& \times \sigma_{e_{M-1}}^{2}(n)=\sigma_{s}^{2}(n),
\end{aligned}
$$

where $\sigma_{e_{M-1}}^{2}(n)=E\left\{e_{M-1}^{2}(n)\right\}$ is the power of the $(M-1)$ th stage error signal. Using the orthogonality of the backward prediction errors in Eq. (3), the terms in the left-hand side of Eq. (13) can be simplified as

$$
\begin{aligned}
2 E\left\{\mathbf{b}^{T}(n)\right. & \left.\Sigma^{-1}(n) \mathbf{b}(n)\right\} \mu(n) \\
& \approx 2 \sum_{i=0}^{M-1} E\left\{b_{i}^{2}(n)\right\} / \eta_{i}(n) \mu(n) \\
& =2 M \mu(n)
\end{aligned}
$$

and

$$
\begin{aligned}
E & \left\{\mathbf{b}^{T}(n) \Sigma^{-1}(n) \mathbf{b}(n) \mathbf{b}^{T}(n) \Sigma^{-1}(n) \mathbf{b}(n)\right\} \mu^{2}(n) \\
& \approx \sum_{i=0}^{M-1} E\left\{b_{i}^{2}(n)\right\} / \eta_{i}(n) \sum_{j=0}^{M-1} E\left\{b_{j}^{2}(n)\right\} / \eta_{j}(n) \mu^{2}(n) \\
& =M^{2} \mu^{2}(n) .
\end{aligned}
$$

In the above simplifications, we used the approximation $E\left\{b_{i}^{2}(n) b_{j}^{2}(n)\right\} \approx E\left\{b_{i}^{2}(n)\right\} E\left\{b_{j}^{2}(n)\right\}$. Hence, Eq. (13) can be rewritten as

$$
\left[1-2 M \mu(n)+M^{2} \mu^{2}(n)\right] \sigma_{e_{M-1}}^{2}(n)=\sigma_{s}^{2}(n) .
$$

By solving Eq. (16), we obtain the variable step-size parameter at time $n$, as given by

$$
\mu_{v s s}(n)=\frac{1}{M}\left[1-\sqrt{\frac{\sigma_{s}^{2}(n)}{\sigma_{e_{M-1}}^{2}(n)}}\right] .
$$

This variable step-size parameter replaces the step-size parameters in Eqs. (4) and (5). In general, the power of
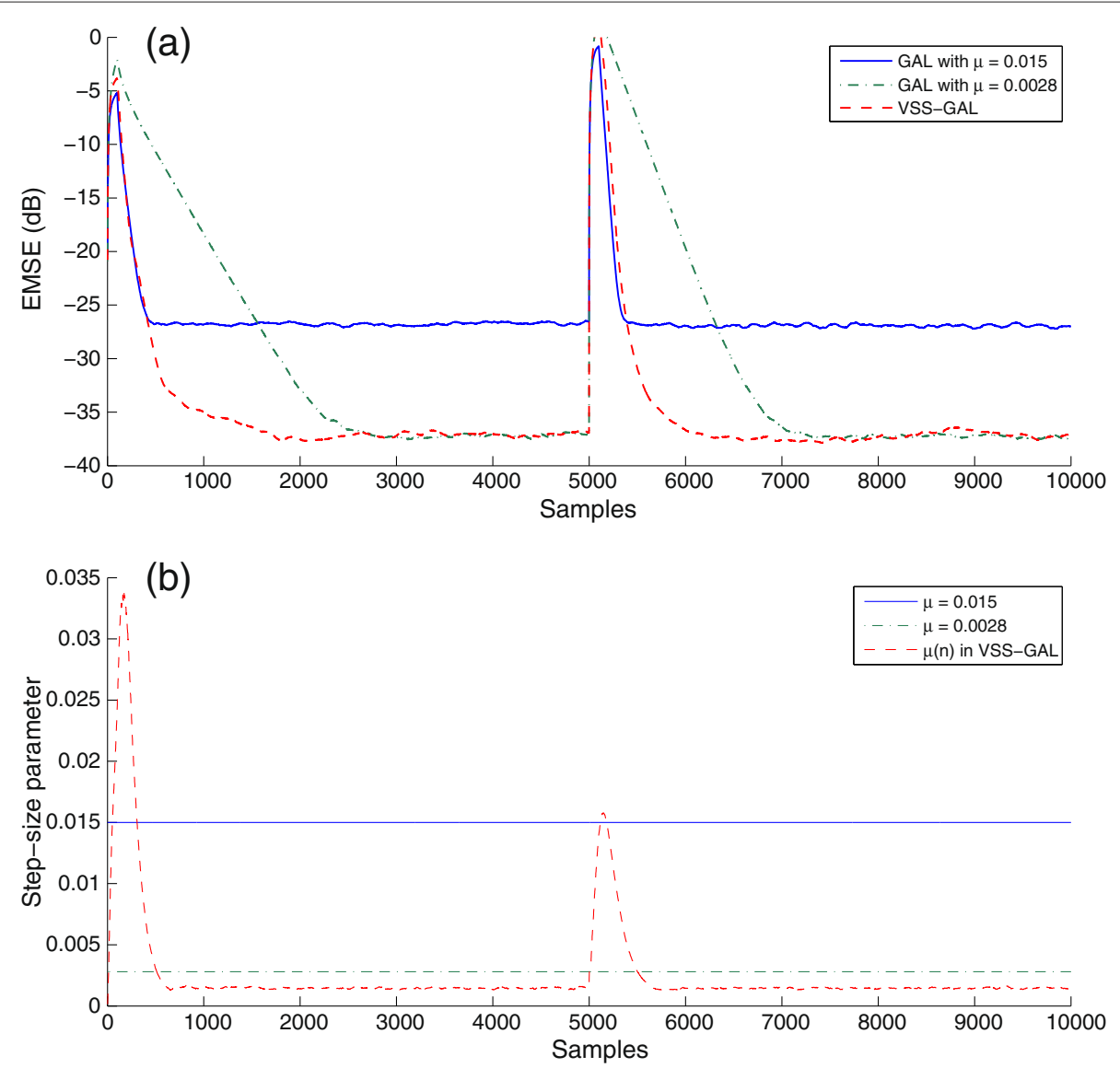

Figure 3 EMSE and step-size parameter of the GAL algorithm and VSS-GAL algorithm. (a) EMSE and (b) step-size parameter of the GAL algorithm with two different step-size parameters, $\mu=0.015$ and $\mu=0.0028$, and the VSS-GAL algorithm, $\lambda_{2}=0.993$. 


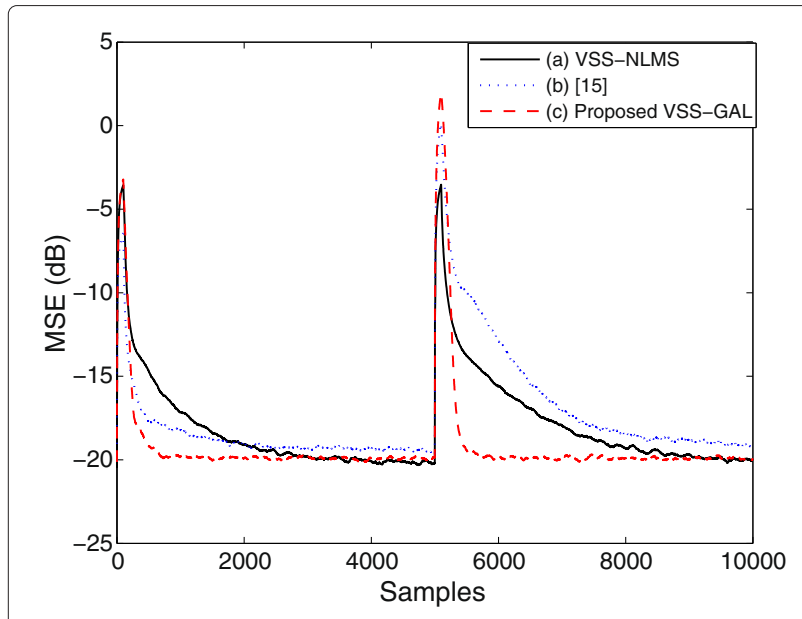

Figure 4 MSE comparison among (a) the VSS-NLMS algorithm, (b) [15], and (c) the proposed VSS-GAL algorithm.

the interference in the sonar signal is much stronger than that of the target signal. Thus, at the initial state, we have $\sigma_{e_{M-1}}^{2}(n) \gg \sigma_{s}^{2}(n)$, and the step-size parameter will be determined as $\mu_{\text {vss }}(n) \approx 1 / M$, which leads to fast convergence. When the algorithm approaches the steady-state, we can expect that $\sigma_{e_{M-1}}^{2}(n) \approx \sigma_{s}^{2}(n)$, and as a result, a fairly small step-size parameter will be used. Thus, narrow notch bandwidths will be attained.

\subsection{Convergence analysis}

The mean square behavior of the VSS-GAL algorithm can be analyzed by evaluating second-order moments of the regression coefficient errors. Suppose that the primary signal is modeled as

$$
d(n)=s(n)+\mathbf{w}^{o T} \mathbf{b}(n),
$$

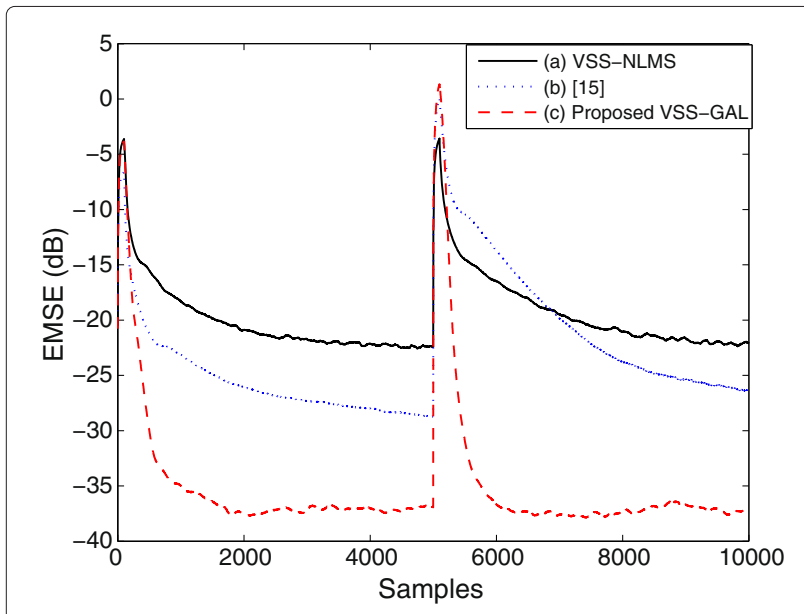

Figure 5 EMSE comparison among (a) the VSS-NLMS algorithm, (b) [15], and (c) the proposed VSS-GAL algorithm. where $\mathbf{w}^{o}=\left[w_{0}^{o}, \ldots, w_{M-1}^{o}\right]^{T}$ is the impulse response of the unknown system with length $M$. The update equation in Eq. (5) can thus be written in terms of the $m$ th stage regression coefficient error, $\tilde{w}_{m}(n)=w_{m}^{o}-w_{m}(n)$, as

$$
\tilde{w}_{m}(n)=\tilde{w}_{m}(n-1)-\frac{\mu(n)}{\eta_{m}(n)} b_{m}(n) e_{m}(n) .
$$

Then, we have

$$
\begin{aligned}
E\left\{\tilde{w}_{m}^{2}(n)\right\}= & E\left\{\tilde{w}_{m}^{2}(n-1)\right\}-2 \mu(n) \\
& \times E\left\{\tilde{w}_{m}(n-1) b_{m}(n) e_{m}(n) / \eta_{m}(n)\right\} \\
& +\mu^{2}(n) E\left\{b_{m}^{2}(n) e_{m}^{2}(n) / \eta_{m}^{2}(n)\right\} .
\end{aligned}
$$

Using the orthogonality of the backward prediction errors in Eq. (3) and the independence condition [16], the second and third terms in the right-hand side of the above equation can be approximated, respectively, as

$$
\begin{aligned}
2 \mu(n) E\left\{\tilde{w}_{m}(n-1) b_{m}(n) e_{m}(n) / \eta_{m}(n)\right\} \\
\approx 2 \mu(n) E\left\{\tilde{w}_{m}^{2}(n-1) b_{m}^{2}(n)\right\} / \eta_{m}(n) \\
\approx 2 \mu(n) E\left\{\tilde{w}_{m}^{2}(n-1)\right\}
\end{aligned}
$$

and

$$
\begin{aligned}
\mu^{2}(n) E\{ & \left.b_{m}^{2}(n) e_{m}^{2}(n) / \eta_{m}^{2}(n)\right\} \\
& \approx \mu^{2}(n) E\left\{\tilde{w}_{m}^{2}(n-1) b_{m}^{4}(n)\right\} / \eta_{m}^{2}(n) \\
& \approx \mu^{2}(n) E\left\{\tilde{w}_{m}^{2}(n-1)\right\}
\end{aligned}
$$

where $\sigma_{e_{m}}^{2}(n)=E\left\{e_{m}^{2}(n)\right\}$ is the power of the $m$ th stage error signal. In the above equation, the same approximation is used in Eq. (15). Using Eqs. (21) and (22), Eq. (20) can be rewritten as

$$
E\left\{\tilde{w}_{m}^{2}(n)\right\}=\left[1-2 \mu(n)+\mu^{2}(n)\right] E\left\{\tilde{w}_{m}^{2}(n-1)\right\} .
$$

Thus, we readily see that $E\left\{\tilde{w}_{m}^{2}(n)\right\}-E\left\{\tilde{w}_{m}^{2}(n-1)\right\}<$ 0 is achieved if the variable step-size parameter $\mu(n)$ is bounded as

$$
0<\mu(n)<1 .
$$

Since the filter order $M$ should be twice as many as the number of sinusoids for sinusoidal interference cancelation, the variable step-size parameter in Eq. (17) always lies within the stability bound.

Next, we will show that the VSS-GAL algorithm recovers the target signal power at the filter output. Substituting Eq. (18) into Eq. (9), we have

$$
e_{M-1}(n)=s(n)+\tilde{\mathbf{w}}^{T}(n-1) \mathbf{b}(n),
$$


where $\tilde{\mathrm{w}}(n)=\left[\tilde{w}_{0}(n), \ldots, \tilde{w}_{M-1}(n)\right]^{T}$. Squaring Eq. (25) and taking expectations, we obtain

$$
\sigma_{e_{M-1}}^{2}(n)=\sigma_{s}^{2}(n)+E\left\{\tilde{\mathrm{w}}^{T}(n-1) \mathbf{b}(n) \mathbf{b}^{T}(n) \tilde{\mathrm{w}}(n-1)\right\} .
$$

Again, using the orthogonality of the backward prediction errors and the independence assumption [16], Eq. (26) can be rewritten as

$$
\sigma_{e_{M-1}}^{2}(n)=\sigma_{s}^{2}(n)+\operatorname{tr}\{\Sigma(n) \mathbf{K}(n-1)\},
$$

where $\mathbf{K}(n-1)=E\left\{\tilde{\mathbf{w}}(n-1) \tilde{\mathrm{w}}^{T}(n-1)\right\}$. If the step-size parameter is properly bounded, as in Eq. (24), we can achieve $E\left\{\tilde{w}_{m}^{2}(\infty)\right\}=0$. Then, we also have $\operatorname{tr}\{\Sigma(\infty) \mathbf{K}(\infty)\}=0$. Thus, the variable step-size parameter in Eq. (17) guarantees that

$$
\lim _{n \rightarrow \infty} \sigma_{e_{M-1}}^{2}(n)=\sigma_{s}^{2}(n)
$$

Thus, it has been proven that the VSS-GAL algorithm in steady-state recovers the target signal power at the final stage of the lattice filter.

\section{Parameter estimation}

To determine the variable step-size parameter $\mu_{\nu s s}(n)$, we need a target signal power $\sigma_{s}^{2}(n)$, which is not available in real-world applications. To solve this problem, we approximate Eq. (26) as

$$
\sigma_{e_{M-1}}^{2}(n) \approx \sigma_{s}^{2}(n)+\tilde{\mathrm{w}}^{T}(n-1) \Sigma(n) \tilde{\mathrm{w}}(n-1) .
$$

The cross-correlation between the backward prediction errors vector and the $(M-1)$ th stage error signal is given by

$$
\mathbf{r}(n)=E\left\{\mathbf{b}(n) e_{M-1}(n)\right\}=\Sigma(n) \tilde{\mathbf{w}}(n-1) .
$$

Now, the target signal power $\sigma_{s}^{2}(n)$ can be estimated by substituting Eq. (30) into Eq. (29):

$$
\sigma_{s}^{2}(n) \approx \sigma_{e_{M-1}}^{2}(n)-\mathbf{r}^{T}(n) \Sigma^{-1}(n) \mathbf{r}(n) .
$$

In practice, $\xi_{m}(n), \eta_{m}(n), \sigma_{e_{M-1}}^{2}(n)$, and $\mathbf{r}(n)$ can be estimated using a single-pole low-pass filter. Without a loss of generality, a variable smoothing factor $\lambda_{1}(n)=$ $1-\mu_{v s s}(n)$ can be used. However, $\mu_{v s s}(n)$ is not available until these parameters are obtained. Thus, we use the previous variable step-size parameter $\mu_{v s s}(n-1)$ to estimate parameters $\xi_{m}(n)$ and $\eta_{m}(n)$, with $\mu_{v s s}(-1)=1 / M$. On the other hand, $\sigma_{e_{M-1}}^{2}(n)$ and $\mathbf{r}(n)$ are estimated using a fixed smoothing factor $0<\lambda_{2}<1$. The following equations denote the estimators.

$$
\begin{aligned}
& \hat{\xi}_{m}(n)=\lambda_{1} \hat{\xi}_{m}(n-1)+\left(1-\lambda_{1}\right)\left\{b_{m}^{2}(n)+f_{m}^{2}(n)\right\} \\
& \hat{\eta}_{m}(n)=\lambda_{1} \hat{\eta}_{m}(n-1)+\left(1-\lambda_{1}\right) b_{m}^{2}(n) \\
& \hat{\sigma}_{e_{M-1}}^{2}(n)=\lambda_{2} \hat{\sigma}_{e_{M-1}}^{2}(n-1)+\left(1-\lambda_{2}\right) e_{M-1}^{2}(n) \\
& \hat{\mathrm{r}}(n)=\lambda_{2} \hat{\mathrm{r}}(n-1)+\left(1-\lambda_{2}\right) \mathbf{b}(n) e_{M-1}(n)
\end{aligned}
$$

The estimate $\hat{\sigma}_{e_{M-1}}^{2}(n)$ can be smaller than $\hat{\sigma}_{s}^{2}(n)$, which can result in negative $\mu_{v s s}(n)$. To avoid this, we use the absolute value of Eq. (17). Hence, the variable step-size parameter is determined as

$$
\mu_{v s s}(n)=\frac{1}{M}\left|1-\sqrt{\left|1-\frac{\hat{\mathbf{r}}^{T}(n) \hat{\Sigma}^{-1}(n) \hat{\mathbf{r}}(n)}{\hat{\sigma}_{e_{M-1}}^{2}(n)}\right|}\right|
$$

\section{Simulation results}

Computer simulations were conducted to evaluate the proposed VSS-GAL algorithm. In sonar signal processing, both the target signal $s(n)$ and interference source $v(n)$ consist of multiple sinusoids and ambient noise $\phi(n)$. To simulate this, we express the target signal and interference source as

$$
\sum_{i=1}^{I} A_{i} \sin \left(2 \pi f_{i} n+\theta_{i}\right)+\phi(n),
$$

where $I$ is the number of the sinusoids and $A_{i}, f_{i}$, and $\theta_{i}$ are the amplitude, frequency, and phase of the $i$ th sinusoid, respectively. We assumed that the interference $v_{1}(n)$ and reference signal $v_{2}(n)$ had sinusoidal components with identical frequencies but different amplitudes and phases. In all simulations, the sampling rate was $f_{s}=8$ $\mathrm{kHz}$, the interference source comprised ten sinusoids of frequencies 250, 630, 1, 020, 1, 380, 1, 890, 2, 100, 2, 530, 2, 950, 3, 460, and 3, $700 \mathrm{~Hz}$. The target signal comprised ten sinusoids of frequencies $280,680,1,080,1,500,1,800$, $2,150,2,830,3,100,3,500$, and $3,730 \mathrm{~Hz}$ and the ambient noise which was an $\mathrm{AR}(2)$ process with the transfer function $1 /\left(1-0.9 z^{-1}+0.3 z^{-2}\right)$. Figure 2 shows power spectral densities of the reference and primary signals where the interference-to-signal ratio (ISR) is $20 \mathrm{~dB}$.

Performance was measured using the mean square error (MSE) defined as $\operatorname{MSE}(n)=E\left\{e_{M-1}^{2}(n)\right\}$ and the excess mean square error (EMSE) defined as $\operatorname{EMSE}(n)=$ $E\left\{\left(e_{M-1}(n)-s(n)\right)^{2}\right\}$ [17]. We measured the EMSE to show how closely the algorithm recovers the target signal at the error output. All the MSE and EMSE were obtained by averaging 100 independent trials.

In Figure 3, the EMSE of the proposed VSS-GAL algorithm is compared with those of the conventional GAL algorithm obtained using two different step-size parameters, $\mu=0.015$ and $\mu=0.0028$. Reference 


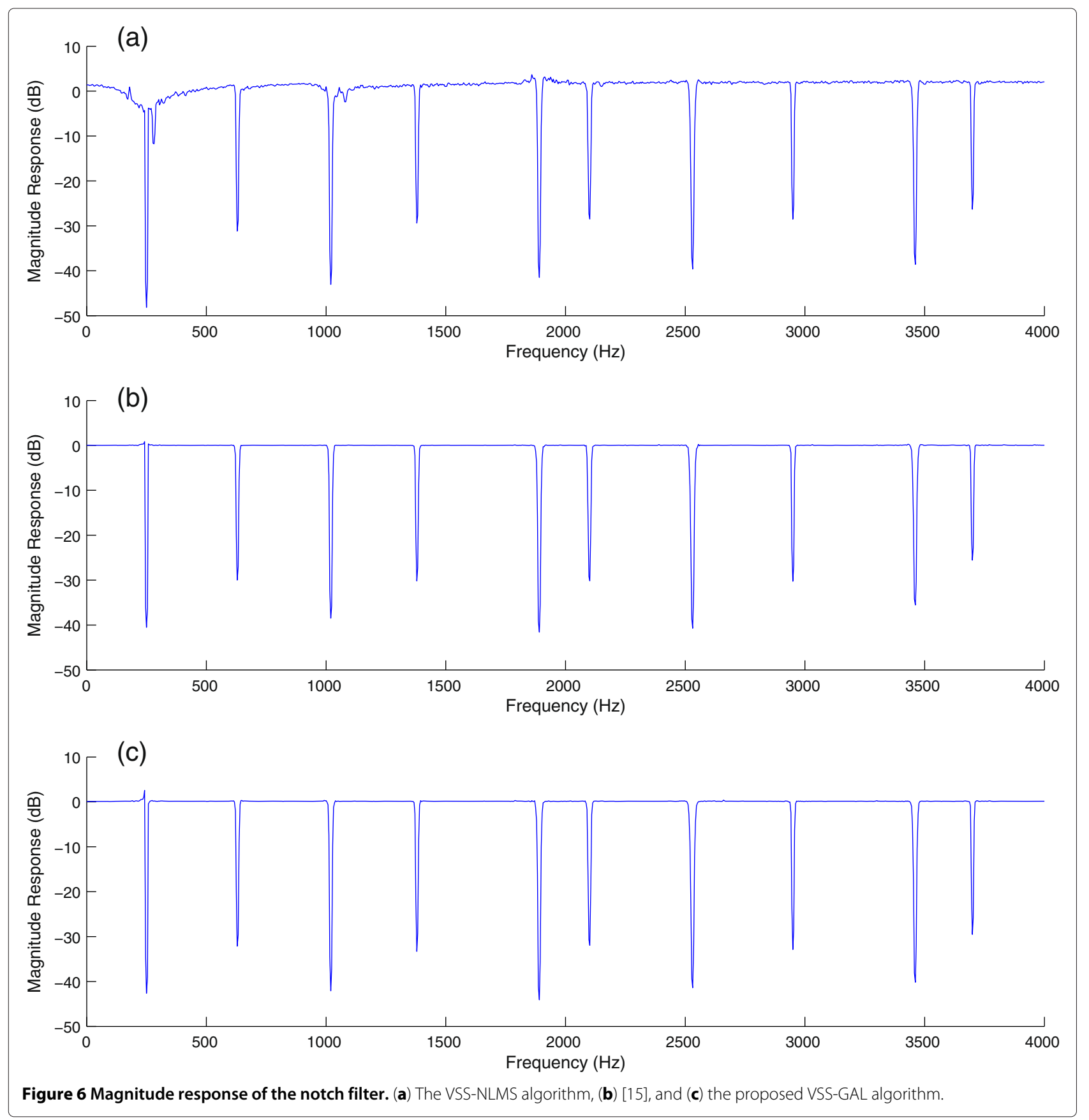

and primary signals shown in Figure 2 were used, and the filter order was $M=20$. To assess tracking ability, the phases of the interference sinusoids were randomly changed at the 5,000th sample. The results in the figure show that the convergence rate of the proposed algorithm is as fast as that of the conventional GAL algorithm with $\mu=0.015$ and the steady-state EMSE is as low as the one obtained using $\mu=0.0028$. We also show the averaged step-size parameter of the VSS-GAL algorithm in Figure 3, which clearly indicates that the proposed VSS scheme desirably adjusts the step-size parameter according to the state of the filter response.

In Figures 4, 5, 6, and 7, we compared the performance of the proposed VSS-GAL algorithm with those of the VSS-NLMS algorithm in [9] and the VSS-GAL algorithm in [15]. Simulation environments were the same as Figure 3. Figure 4 shows the MSE curves. It is shown that the proposed VSS-GAL algorithm converges faster than the VSS-NLMS algorithm, and the steady-state MSE 


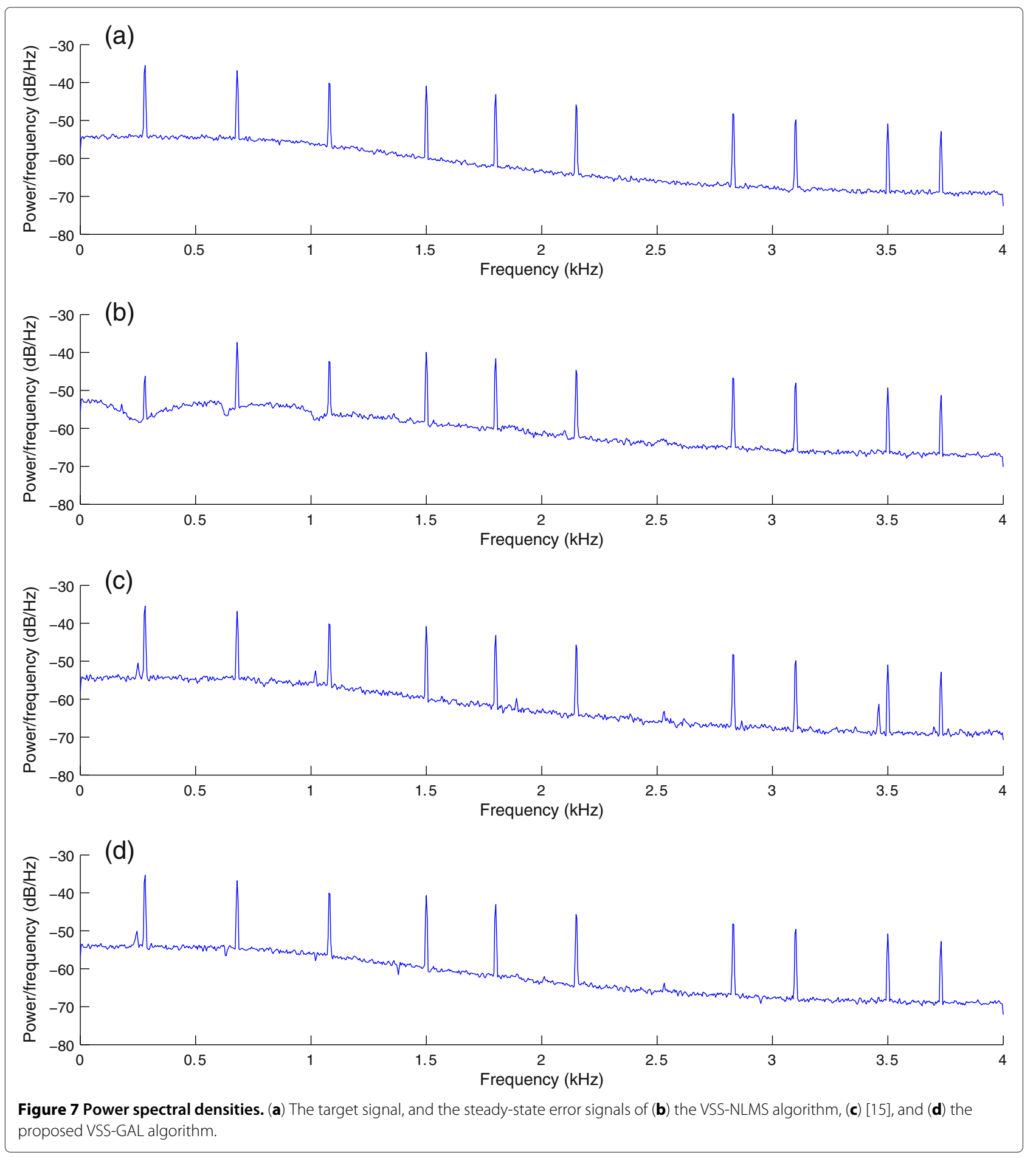

of the proposed VSS-GAL algorithm is slightly higher than that of the VSS-NLMS algorithm. The previous VSS-GAL algorithm in [15], on the other hand, shows slow convergence especially when the interference was changed at 5,000 samples. The reason is that, since it requires an assumption that the adaptive filter has converged well enough to estimate the target signal power, the estimated parameters during the transient state are biased ones. The EMSE curves in Figure 5 more clearly demonstrate the superiority of the proposed VSS-GAL algorithm over the other algorithms. Figure 5 shows that the proposed VSS-GAL algorithm produces significantly lower steady-state EMSE than both the VSS-NLMS and the previous VSS-GAL algorithms, which indicates that 
Table 1 Steady-state performance comparison of algorithms for ISR

\begin{tabular}{|c|c|c|c|c|c|c|}
\hline \multirow{2}{*}{$\begin{array}{l}\text { ISR }(\mathrm{dB}) \\
\text { Performance measure }(\mathrm{dB})\end{array}$} & \multicolumn{2}{|c|}{10} & \multicolumn{2}{|c|}{20} & \multicolumn{2}{|c|}{30} \\
\hline & MSE & EMSE & MSE & EMSE & MSE & EMSE \\
\hline VSS-NLMS & -10.33 & -14.35 & -20.33 & -22.85 & -30.11 & -31.39 \\
\hline$[15]$ & -9.95 & -30.56 & -19.82 & -34.10 & -29.00 & -35.72 \\
\hline Proposed VSS-GAL & -9.94 & -32.28 & -19.93 & -41.00 & -29.60 & -40.58 \\
\hline
\end{tabular}

the proposed VSS-GAL algorithm is able to recover the target signal with much lower distortion than the other algorithms. This is because the proposed VSS-GAL algorithm produces accurate notches with narrow bandwidths which are independent of the amplitudes of sinusoidal interferences and proportionate only to the step-size parameter.

In [5], it was shown that the $i$ th notch bandwidth of the NLMS transversal filter for multiple sinusoidal interference can be approximated as

$$
B W_{N L M S}^{i}=\tilde{\mu} M A_{i}^{2} f_{s} / 2 \pi(\mathrm{Hz}),
$$

where $\tilde{\mu}=\mu / \sigma_{x}^{2}$ is the normalized step-size parameter and $\sigma_{x}^{2}$ is the power of the reference signal. Thus, the notch bandwidth of the NLMS transversal filter is proportional to the amplitude of the sinusoidal interference $A_{i}$ and the filter order $M$. On the other hand, the $i$ th notch bandwidth of the GAL filter can be approximated as [5]

$$
B W_{G A L}^{i}=\mu f_{s} / \pi(\mathrm{Hz}),
$$

where the notch bandwidth is independent of the amplitude of the sinusoidal interference and it is proportional only to the step-size parameter $\mu$. In the VSS scheme, a small step-size parameter is generally used at the steadystate so that narrow notches can be provided.

To compare the notch behaviors, we evaluated the transfer function from $d(n)$ to $e(n)$ at the steady state. The results are presented in Figure 6. Also, the power spectral densities of the recovered target signals are presented in Figure 7. The transfer functions in Figure 6 show that the VSS-NLMS algorithm distorts the frequency response around the frequencies of sinusoidal interferences with large amplitudes, which is clearly visible at
250 and 1,020 Hz. As a result, the VSS-NLMS algorithm produced high EMSE. On the other hand, both the previous and the proposed VSS-GAL algorithms produced insignificant distortions at frequencies other than the interference frequencies. However, the notches produced by the previous VSS-GAL algorithm were not sufficiently accurate so that the interferences were not completely removed, which can be seen from the steady-state power spectral density in Figure 7(b). Consequently, the proposed VSS-GAL algorithm achieved the lowest EMSE among the algorithms, and the target signal was recovered with the smallest distortion.

Finally, to confirm the performance in various environments, we measured the steady-state MSE and EMSE according to the ISR and filter order. The results are summarized in Tables 1 and 2. EMSE results indicate that the proposed VSS-GAL algorithm recovers the target signal power with much less distortions than both the VSS-NLMS algorithm and the VSS-GAL algorithm in [15].

\section{Conclusions}

We proposed a variable step-size scheme for the GAL algorithm for cancelation of sinusoidal interference. The proposed VSS scheme was designed to recover the target signal from within the error signal. Simulation results showed that the proposed algorithm achieved a fast convergence rate, good tracking ability, and low steady-state EMSE. Compared to the VSS-NLMS algorithm, it formed narrow notches at the interference frequencies, so it could recover the target spectrum with significantly smaller distortions than the VSS-NLMS algorithm.

\begin{tabular}{|c|c|c|c|c|c|c|}
\hline \multirow{2}{*}{$\begin{array}{c}\text { Filter order } \\
\text { Performance measure }(\mathrm{dB})\end{array}$} & \multicolumn{2}{|c|}{10} & \multicolumn{2}{|c|}{20} & \multicolumn{2}{|c|}{30} \\
\hline & MSE & EMSE & MSE & EMSE & MSE & EMSE \\
\hline VSS-NLMS & -6.91 & -6.97 & -20.33 & -22.85 & -20.32 & -22.86 \\
\hline$[15]$ & -10.75 & -11.92 & -19.82 & -34.10 & -19.86 & -35.55 \\
\hline Proposed VSS-GAL & -13.12 & -14.11 & -19.93 & -41.00 & -19.90 & -38.68 \\
\hline
\end{tabular}

Table 2 Steady-state performance comparison of algorithms for filter order 


\begin{abstract}
Abbreviations
ANC, Adaptive noise cancelation; EMSE, Excess mean square error; GAL, Gradient adaptive lattice; ISR, Interference-to-signal ratio; MSE, Mean square error; NLMS, Normalized least mean square; VSS, Variable step size; VSS-GAL, Variable step-size gradient adaptive lattice; VSS-NLMS, Variable step-size normalized least mean square.
\end{abstract}

\section{Competing interests}

The authors declare that they have no competing interests.

\section{Author details}

${ }^{1}$ School of Electrical and Electronic Engineering, Yonsei University, Seoul 120-749, South Korea. ${ }^{2}$ Computer and Telecommunications Engineering

Division, Yonsei University, Wonju, Kangwon 220-710, South Korea.

Received: 2 November 2012 Accepted: 15 February 2013

Published: 16 May 2013

\section{References}

1. GA Beattie, PA Cotterill, Assessing performance: submarine flank arrays. Sea Technol. 36, 45-49 (1995)

2. B Widrow et al, Adaptive noise cancelling: principles and applications. Proc. IEEE. 63, 1692-1716 (1975)

3. JR Glover, Adaptive noise cancelling applied to sinusoidal interferences. IEEE Trans. Acoust., Speech, Signal Process. 25(6), 484-491 (1977)

4. S Haykin, Adaptive Filter Theory, 4th ed. (Prentice-Hall, Upper Saddle River, 2002)

5. RC North, JR Zeidler, TR Albert, WH Ku, in Acoustics, Speech, and Signal Processing, 1992 ICASSP-92. 1992 IEEE International Conference on Comparison of adaptive lattice filters to LMS transversal filters for sinusoidal cancellation (San Francisco, 1992)

6. LJ Griffiths, in Acoustics, Speech, and Signal Processing, IEEE International Conference on ICASSP'78. An adaptive lattice structure for noise-cancelling applications (Tulsa, 1978)

7. EH Satorius, JD Smith, PM Reeves, in Acoustics, Speech, and Signal Processing, IEEE International Conference on ICASSP'79. Adaptive noise cancelling of a sinusoidal interference using a lattice structure (Washington DC, 1979)

8. HC Shin, AH Sayed, WJ Song, Variable step-size NLMS and affine projetion algorithms. IEEE Signal Process. Lett. 11(2), 132-135 (2004)

9. J Benesty, H Rey, LR Vega, S Tressens, A nonparametric VSS NLMS algorithm. IEEE Signal Process. Lett. 13(10), 581-584 (2006)

10. C Paleologu, S Ciochina, J Benesty, Variable step-size NLMS algorithm for under-modeling acoustic echo cancellation. IEEE Signal Process. Lett. 15(10), 5-8 (2008)

11. C Paleologu, J Benesty, S Ciochină, A variable step-size affine projection algorithm designed for acoustic echo cancellation. IEEE Trans. Audio, Speech, Lang. Process. 16(8), 1466-1478 (2008)

12. Y Zhang, N Li, JA Chambers, Y Hao, New gradient-based variable step size LMS algorithms. EURASIP J. Adv. Signal Process (2008). doi:10.1155/2008/529480

13. L Liu, M Fukumoto, S Saiki, S Zhang, A variable step-size proportionate affine projection algorithm for identification of sparse impulse response, EURASIP J. Adv. Signal Process (2009). doi:10.1155/2009/150914

14. SE Abadi, A Arani, A family of variable step-size affine projection adaptive filter algorithms using statiscs of channel impulse response. EURASIP J. Adv. Signal Process (2011). doi:10.1186/1687-6180-2011-97

15. SW Kim, YC Park, DH Youn, in Acoustics, Speech and Signal Processing (ICASSP) 2012 IEEE International Conference on. A variable step-size filtered-x gradient adaptive lattice algorithm for active noise control (Kyoto, 2012)

16. S Makino, Y Kaneda, N Koizumi, Exponentially weighted step-size NLMS adaptive filter based on the statistics of a room impulse response. IEEE Trans. Speech Audio Process. 1, 101-108 (1993)

17. JM Gorriz, J Ramirez, S Cruces-Alvarez, CG Puntonet, A novel LMS algorithm applied to adaptive noise cancellation. IEEE Signal Process. Lett. 16(1), 34-37 (2009)

doi:10.1186/1687-6180-2013-106

Cite this article as: Kim et al:: A variable step-size gradient adaptive lattice algorithm for multiple sinusoidal interference cancelation. EURASIP Journal on Advances in Signal Processing 2013 2013:106

\section{Submit your manuscript to a SpringerOpen ${ }^{\mathcal{O}}$ journal and benefit from:}

- Convenient online submission

- Rigorous peer review

- Immediate publication on acceptance

- Open access: articles freely available online

- High visibility within the field

- Retaining the copyright to your article

Submit your next manuscript at $>$ springeropen.com 